\title{
Eye-movement patterns during visual information processing
}

JOHN D. GOULD AND AMY SCHAFFER

IBM RESEARCH CENTER, YORKTOWN HEIGHTS, NEW YORK

\begin{abstract}
Eye-movement patterns were recorded while Ss visually scanned 5-cell patterns to compare the sum of the 3 digits in each of 4 peripheral cells with the sum of the 3 digits in the central "target" cell.Both larger target sums and greater target-non-target similarity caused significantly longer fixations; number and pattern of fixations were independent of experimental variables. Results supported predictions based upon previous studies correlating eye-movement patterns and visual stimuli.
\end{abstract}

\section{Problem}

Since little or no perception occurs between points of fixation, the eye-movement parameters relevant for visual perception are number, duration, and location of eye fixations. One purpose of an on-going series of studies in which subjects (Ss) scan stimulus matrices to determine the number of "targets" on each matrix has been to assess the changes in these eye-movement parameters as a function of differences within and between classes of stimuli. From this point of view three types of experiments were designed, differing in rank order on the amount of perceptual discrimination, cognitive activity, or perceptual-analytic "tests" needed to recognize a "target."

In the simplest case Ss scanned 6 by 6 numeric matrices, each cell containing a single digit, and it was found that average number of fixations increased with increases in target digit frequency from 0-7, even though each cell had to be perceived regardless of target frequency (Gould \& Schaffer, 1965a). No change in duration of fixation occurred. When peripheral vision was reduced, however, by using a 3 by 3 matrix subtending the same $16^{\circ}$ angle, number of fixations was not influenced by target digit frequency (Gould \& Schaffer, 1965b).

In a more difficult task (Gould \& Schaffer, 1965b), stimuli consisting of patterns were presented in approximately the same 3 by 3 matrix. Again no changes in eye-movement parameters were found with target frequency, but as target-non-target similarity increased both the average number and average duration of fixations increased significantly. These two increases were due to more and longer looks at non-targets only. Average duration of looks at target patterns, although significantly longer than looks at non-targets, was independent of pattern similarity.

The increased duration of fixation on non-targets as a function of similarity was interpreted as possibly reflecting additional visual-perceptual or higher order "tests" needed to discriminate a non-target from a highly similar target pattern. The fact that fixations were significantly longer on target patterns than nontargets suggested that more visual "tests" were needed to detect an exact match between the memorized target pattern and a given fixated pattern than to detect a difference.

Two hypotheses were developed based on these results. First, as types of stimuli to be scanned and processed become more complex and demand increasing amounts of cognition, duration of fixation becomes more variable compared to number of fixations. Second, in the pattern experiment looks at targets were longer than at non-targets because all targets were exactly the same physically (appeared exactly the same) as the memorized target stimulus, thus necessitating a longer, more complete set of visual "tests" to be made on a given fixated pattern if it was, in fact, a target than if it had been a non-target. Assuming the second hypothesis, we would predict that if target stimuli were not exactly the same physically, but were alike at a more abstract level, e.g. their differences, sums, ratios, etc., then little difference between duration of fixation on targets and non-targets would occur.

The present experiment tested these two hypotheses by (1) utilizing a task that required a greater amount of cognition; and (2) allowing the physical stimuli (digits) for targets to vary but requiring their sums to equal the same total as the memorized target total.

\section{Method}

Eye movements of six volunteer college Ss were recorded on film (22 frames/sec.) using a cornealreflectance, television eye-marker system (Mackworth \& Mackworth, 1958), while they scanned and added up three digits in each corner of a $16^{\circ}$ stimulus to determine how many corners equalled the sum of the three "target" digits in the center of the stimulus. The general methodology of recording and data analyses in these experiments is described more fully elsewhere (Gould \& Schaffer, 1965a, 1965b).

The Sum of the three digits composing a target was 8,14 , or 20 . Target-non-target Similarity was varied by setting the average deviation between target and non-target Sums at 2, 5, or 8. Frequency of targets was varied 0-4. Each $S$ went through two different blocks of the 45 conditions ( 3 levels of Sums by 3 of Similarity by 5 of Frequency), with eye-movement records being obtained on the second block. 


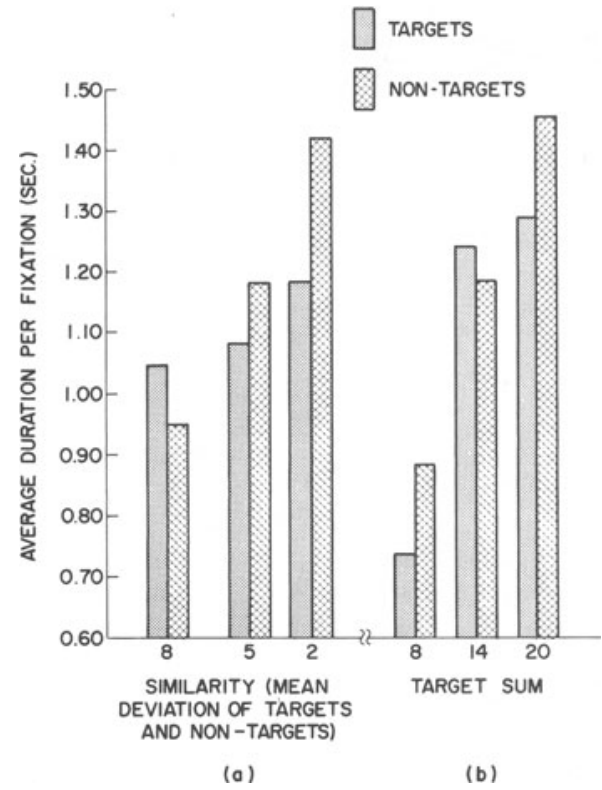

Fig. 1. Average duration per fixation as a function of (a) the similarity or average deviation of target and non-target totals; (b) the arithmetic size of the target Sum. Average duration per fixation on target digits was measured for each trial by programming the computer to determine the total time (in camera frames) that fixations occurred on targets and dividing this by the number of target fixations for that trial. Mean duration on non-targets was computed similarly.

\section{Results}

Increased scan times resulted as target Sums became larger $(p<.001)$, and also as target-non-target Similarity increased $(p<.001)$. These longer scan times were due to an increased average duration per fixation for Similarity $(F=27.36 ; \mathrm{df} 2 / 10 ; \mathrm{p}<.001)$, and also for the Sum variable $(F=54.18 ;$ df $2 / 10 ; p<.001)$. No significant changes in number of fixations on the four corners of the matrix occurred $(\mathrm{Mean}=5.2 ; \mathrm{SD}=1.10$ fixations).

As shown in Fig. 1a, average duration per fixation on non-targets $(\mathrm{F}=27.14 ;$ df $2 / 10 ; \mathrm{p}<.001)$, as well as on targets $(F=4.84 ;$ df $2 / 10 ; p<.05)$, increased significantly with increased Similarity. No overall difference occurred between the duration of looks at targets and non-targets $(F<1.0)$. When targets were least similar to non-targets, however, Ss looked longer at the target digits. With increased similarity Ss looked longer, presumably took longer to process, the non-target digits (i.e., a significant Target-non-target by Similarity interaction; $\mathrm{F}=6.23$; df $2 / 10 ; \mathrm{p}<.05$ ).

Figure $1 \mathrm{~b}$ shows that both duration of fixation on targets $(F=36.37 ;$ df $2 / 10 ; p<.001)$ and also on nontargets $(F=13.52 ;$ df $2 / 10 ; p<.001)$ increased as target Sums became larger. No significant interaction was found. In regard to the Frequency variable, average duration per fixation was generally longer for stimuli containing more targets $(F=4.82 ;$ df $4 / 20 ; p<.001$; Means $=.97,1.04,1.13,1.20$, and $1.12 \mathrm{sec} .$, respectively, for Frequency $=0-4$ ).

\section{Diseussion}

The two main results of this experiment relevant to the predicted hypotheses are, first, that duration, and not number, of fixations varied significantly as the task became more difficult; and secondly, that no difference between the duration of fixations on targets and non-targets occurred.

The first finding, taken in conjunction with our previous experiments on similar scanning tasks supports the notion that duration of looks is affected relatively more than number of looks as the amount of cognitive activity associated with the task increases. This fact suggests, for any precise informationprocessing model relating these two parameters of visual input, that their relationship is not necessarily multiplicative, as has been suggested for continuous tasks (Senders, 1964).

The finding of no difference between duration of fixation on target versus non-target digit sets tends to support the explanation offered above in connection with a previous study using patterned stimuli, i.e., that the longer duration of fixation on target patterns relative to non-target patterns was due to all target patterns on a given stimulus being physically the same. It should be noted, however, that only four, instead of eight, cells were scanned in the present study.

Lorens \& Darrow (1962) reported a positive relation between frequency of eye fixations and ability to solve mental arithmetic problems. The present experiment indicates, however, that the number of gross eye fixations are nearly minimal when such problems are presented visually (rather than aurally).

All Ss' patterns of eye movements in this rather structured task were quite similar in that each $\mathrm{S}$ first fixated the center cell and then the top row followed by the bottom row. These patterns remained relatively stable regardless of the number of targets $S$ had already perceived during that trial. Various applications of conditional probability measures, based upon the number of targets previously perceived within a trial, failed to describe the pattern of fixations as well as the simpler description above.

\section{Heferences}

Gould, J. D., \& Schaffer, A. Eye-movement patterns in scanning numeric displays. Percept. mot. Skills, 1965a, 20, 521-535.

Gould, J. D., \& Schaffer, A. Eye movements in visual information processing: Scanning numeric displays and patterned stimuli. In preparation. A portion of these results were presented at E. P. A., Atlantic City, 1965b.

Lorens, S. A., \& Darrow, C. W. Eye movements, EEG, GSR, and EKG during mental multiplication. EEG clin. Neurophys., 1962, $14,739-746$.

Mackworth, J. F., \& Mackworth, N. H. Eye fixations recorded on changing visual scenes by the television eye-marker. J. Opt. Soc. Amer., 1958, 48, 439-445.

Senders, J. W. The human operator as a monitor and controller of multidegree of freedom systems. IEEE trans. hum. fact. Electron., 1964, 5, 1-5. 